\title{
Establishment of the three-dimensional kinematic reference frame by space geodetic measurements
}

\author{
M. D. Gerasimenko ${ }^{1}$ and Teruyuki Kato ${ }^{2}$ \\ ${ }^{1}$ Institute of Applied Mathematics, 690041 Vladivostok, Russia \\ ${ }^{2}$ Earthquake Research Institute, University of Tokyo, Tokyo, Japan
}

(Received October 21, 1999; Revised August 9, 2000; Accepted August 9, 2000)

\begin{abstract}
The purpose of this research is to provide a new algorithm for fixing the three-dimensional kinematic reference frame of space geodetic stations in which only vertical components of quasi-stable site velocities for every $X, Y$, $Z$ direction are used. This KRF does not depend upon any geological model and, thus, is free from the hidden errors coming from the uncertainties of the reference plate motion model, errors in survey data of selected sites as reference stations and the misfit between the measurements and the model predictions. The method has been applied to the VLBI data collected during the period from 1979 to 1997 analyzed by NASA. We have used for the analysis the estimated rate of change of 340 baseline vectors between 59 VLBI sites. As it is clear from our numerous experiments, this algorithm gives a fairly stable results which are in a good agreement with the NUVEL-1 NNR plate motion model if we include for fixing of KRF even a few sites (15-20 points). In the worst case our results obtained using various criteria for selection of quasi-stable sites have $1 \mathrm{~mm} / \mathrm{yr}$ level agreement for all sites in the horizontal and vertical directions. The agreement of our results and the NUVEL-1 NNR model is on the order of a few millimeters per year in each coordinate. The largest discrepancies reach $20 \mathrm{~mm} / \mathrm{yr}$ in the sites close to the plate boundaries. The uncertainties of vertical direction do not exceed $1 \mathrm{~mm} / \mathrm{yr}$ for half of the sites. As a final result, we do not find any clear evidence suggesting the change of Earth's radius, which is considerably less than $1 \mathrm{~mm} / \mathrm{yr}$.
\end{abstract}

\section{Introduction}

Many of the former studies of tectonic plate motion and Earth deformation have been done using the threedimensional kinematic reference frame (KRF) of Very Long Baseline Interferometry (VLBI), Satellite Laser Ranging (SLR) and/or Global Positioning System (GPS) geodetic stations tied to a geological plate motion model. As is well known, very often such an approach leads to rather large changes of the site velocities depending on which system of chosen geodetic sites for KRF was constrained in its estimation (Robbins et al., 1993; Heki, 1996). This is due to the hidden errors coming from the uncertainties of the reference plate motion model, ignoring errors in the survey data of selected sites as reference stations and the misfit between the measurements and the model predictions (Heki, 1996). These effects can be seen in the results of computation made by different authors who are using various approaches for selection of reference stations with fixed velocity components to a priori values. For example, Ma et al. (1994) fixed the horizontal velocity of Westford (Massachusetts) and the change in direction of the vector from Westford to Richmond (Florida) to values predicted by the NUVEL-1 NNR plate motion model (Argus and Gordon, 1991). Additionally they fixed the vertical movements of Westford, Richmond and Kauai (Hawaii) to zero. Fallon and Dillinger (1992) fixed the sum of all velocities and the net angular momentum to

Copy right(c) The Society of Geomagnetism and Earth, Planetary and Space Sciences (SGEPSS); The Seismological Society of Japan; The Volcanological Society of Japan; The Geodetic Society of Japan; The Japanese Society for Planetary Sciences. zero in order to provide a minimally constrained adjustment and then they rotated and translated the network in order to minimize the difference between the geologic model and the geodetic velocities determined by VLBI.

Heki (1996) used 16 reference stations located in stable plate interiors which were defined as areas more than 500 $\mathrm{km}$ from the nearest plate boundary. He applied a small "translation and a rotation for the entire network in a threedimensional space so that the differences in the "horizontal" velocities between the VLBI observations and the model predictions are minimized".

Other authors used in principle the same approaches to establish the KRF: all of them tied to a geologic plate motion model. But any geologic model is based on a geological record spanning the last few million years and does not essentially fully reflect the plate motion at the present instant.

It is possible too that the velocity of a particular site reflects a regular deformation of the underlying plate, especially near a plate boundary. These effects would mean that such a $\mathrm{KRF}$ is not free from plate model errors and depends on the unpredicted movements of plate fixed stations (random and systematic measurement errors). Another question arises from a possible inadvertent bias resulting from a non-uniform station distribution on each plate.

Unlike some other formulations we have proposed an approach to fix the KRF which does not use any geologic plate motion model. We are using only space geodetic data by itself. More than that we are using only vertical components of velocities which do not exceed a small chosen bound. 


\section{Algorithm}

As well known in order to fix the KRF by geodetic data we must solve the set of observation equation

$$
A \delta X+L=V
$$

with the set of constraints

$$
B \delta X=C
$$

where $L$ is a vector of observations (rate of change of baseline vectors), $A$ is a design matrix, $\delta X$ is a vector of unknown velocities for each station of geocentric cartesian coordinate $X, Y, Z$ and $V$ is a residual vector.

The choice of the set of constraints (2) implies the definition of reference frame. In Eq. (2) vector $C$ is a vector of known constants and matrix

$$
B=G^{T} I_{B}
$$

where matrix $G^{T}$ being the known eigen vectors to the zero eigen values of the normal matrix $N=A^{T} P A, P$ is a weight matrix. The matrix $I_{B}$ is a "unit" matrix in which the diagonal elements are equal to zero for those parameters which do not affect the choice of the KRF.

The main shortcomings of such an approach are mentioned in the introduction. In this study we offer to use instead of constraints (2) a constraint

$$
B \delta X_{H}=0
$$

where the vector $\delta X_{H}$ includes only vertical components of vector $\delta X$ and vector $C=0$. But the constraint (4) are inconsistent with Eq. (1). That's why we introduce the unknown matrix $B_{H}$ and transform the Eq. (4) to the form

$$
B_{H} \delta X=0 \text {. }
$$

Now let for $i$-th point of the geodetic net the formula

$$
\delta X_{i}=C_{i}^{T} \delta x_{i}
$$

where

$$
C_{i}^{T}=\left(\begin{array}{ccc}
-\sin B \cos L & -\sin L & \cos B \cos L \\
-\sin B \sin L & \cos L & \cos B \sin L \\
\cos B & 0 & \sin B
\end{array}\right)
$$

is the matrix of transformation from topocentric coordinate changes $\delta x_{i}=\left(d x_{i}, d y_{i}, d H_{i}\right)^{T}$ to geocentric changes $\delta X_{i}=\left(d X_{i}, d Y_{i}, d Z_{i}\right)^{T}, d x_{i}, d y_{i}$ and $d H_{i}$ are the north, east and up component changes, $B$ and $L$ are the geodetic latitude and longitude (here and below the index $i$ is omitted). The Eq. (6) leads to the equation

$$
\delta X_{H, i}=C_{H, i}^{T} \delta x_{i}
$$

where sub-vector $\delta X_{H, i}$ includes only vertical components of sub-vector $\delta X_{i}$ and matrix

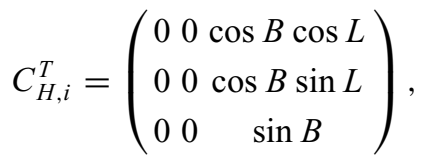

or taking into account (6) we have an equation

$$
\delta X_{H, i}=C_{H, i}^{T} C_{i} \delta X_{i}=S_{i} \delta X_{i}
$$

where matrix $S_{i}=C_{H, i}^{T} C_{i}$. Substituting (8) to Eq. (4) we have the matrix

$$
B_{H}=B S=G^{T} I_{B} S
$$

where the quasi-diagonal matrix $S$ is composed of the blocks $S_{i}$. If for some point we put $S_{i}=I$ (unit matrix), all three $X, Y, Z$ full components of this point will take part in the establishment of the KRF.

The final set of equations can be written as

$$
\begin{gathered}
A \delta X+L=V, \\
B_{H} \delta X=0 .
\end{gathered}
$$

\section{Numerical Example}

The foregoing algorithm has been applied to the VLBI data obtained during the period from 1979 to 1997 analyzed as NASA Goddard Space Flight Center's VLBI terrestrial reference frame solution number 1102g, August 1998 (Ma and Ryan, 1998). This solution gives three sets of rates of change between each pair of two stations under consideration. These quantities are the length, transverse and "vertical" rate of change which were used here in order to obtain three-dimensional KRF of VLBI stations. The combination of the VLBI, SLR and GPS data would improve the accuracy of the KRF but the main aim of our study is only a matter of demonstration of applicability of our algorithm.

The 340 baseline vectors connecting 59 stations were used to calculate station motion. The choice of the quasi-stable points can be found in a process of successive re-adjustment of the network and a subsequent analysis of its results. It should be noted that it is necessary to use as many reference stations as possible in order to dilute the risk of degradation of the results by unexpected movements. We assume that a point of the network is quasi-stable if the change of its height does not exceed a few $\mathrm{mm} / \mathrm{yr}$. We have used $|d H|<5 \mathrm{~mm} / \mathrm{yr}$. Under this condition 45 quasi-stable stations were selected in order to fix the KRF.

Table 1 lists these stations and their topocentric velocities with the NUVEL-1 NNR and VLBI-SLR horizontal velocities obtained by Robbins et al. (1993). The last allows us both to compare our solution and to determine its quality. Unfortunately, our network and the others are not identical. That is why the Table 1 contains not all data but only available for those existent identical stations. The KRF that was adopted by Robbins et al. (1993) is defined by NUVEL-1 NNR modeled horizontal velocities for three stations located on North American plate.

The remaining 14 free stations and their information are listed in Table 2.

Despite of the fact that the Robbins et al. (1993) velocities are not directly comparable to ours because their velocities are derived from essentially another data set and used different processing procedures, we can see fairly close agreement between the two horizontal velocity sets, all of which agree to better than $\sim 8 \mathrm{~mm} / \mathrm{yr}$. The agreement of our solution 
Table 1. Kinematic vectors for quasi-stable VLBI tracking sites (in mm/yr).

\begin{tabular}{|c|c|c|c|c|c|c|c|c|c|c|c|}
\hline \multirow{3}{*}{$\begin{array}{l}\text { Station } \\
\text { number }\end{array}$} & \multirow{3}{*}{$\begin{array}{l}\text { Station } \\
\text { name }\end{array}$} & \multicolumn{6}{|c|}{ VLBI } & \multirow{2}{*}{\multicolumn{2}{|c|}{$\begin{array}{c}\text { NUVEL-1 } \\
\text { NNR }\end{array}$}} & \multirow{2}{*}{\multicolumn{2}{|c|}{$\begin{array}{c}\text { Robbins } \\
\text { et al. }\end{array}$}} \\
\hline & & \multicolumn{2}{|c|}{ North } & \multicolumn{2}{|c|}{ East } & \multicolumn{2}{|c|}{ Up } & & & & \\
\hline & & $d x$ & $\sigma_{d x}$ & $d y$ & $\sigma_{d y}$ & $d H$ & $\sigma_{d H}$ & $d x$ & $d y$ & $d x$ & $d y$ \\
\hline 7282 & ALGOPARK & 4.4 & 1.2 & -17.5 & 0.6 & 3.3 & 0.6 & 3.4 & -18.1 & & \\
\hline 7614 & BR-VLBA & -8.1 & 0.9 & -14.8 & 0.9 & -3.3 & 0.8 & -13.5 & -15.8 & & \\
\hline 7231 & DSS15 & -3.4 & 1.3 & -20.3 & 1.3 & 0.1 & 1.7 & -12.6 & -13.0 & & \\
\hline 1642 & DSS45 & 56.0 & 1.2 & 17.2 & 0.6 & 2.0 & 0.8 & 56.6 & 18.6 & 49.4 & 20.5 \\
\hline 1665 & DSS65 & 18.0 & 1.1 & 18.9 & 0.9 & 2.5 & 0.6 & 16.1 & 19.2 & & \\
\hline 7203 & EFLSBERG & 16.4 & 1.0 & 19.9 & 1.0 & 2.8 & 1.0 & 14.8 & 19.5 & & \\
\hline 7613 & FD-VLBA & -3.0 & 1.1 & -13.4 & 0.8 & -0.6 & 0.7 & -7.5 & -12.7 & & \\
\hline 7297 & FORTLEZA & 13.7 & 0.9 & -5.0 & 0.6 & 1.0 & 1.2 & 12.0 & -5.7 & & \\
\hline 7225 & GILCREEK & -20.7 & 0.5 & -11.1 & 1.0 & 2.7 & 0.9 & -21.4 & -11.0 & & \\
\hline 1513 & GOLDVENU & -1.7 & 1.3 & -19.0 & 1.2 & 3.1 & 2.0 & -12.5 & -12.9 & & \\
\hline 7102 & GOFR7102 & 9.2 & 1.9 & -14.9 & 1.8 & -3.9 & 4.5 & 3.9 & -16.0 & & \\
\hline 7232 & HARTRAO & 18.4 & 0.8 & 18.6 & 1.0 & -0.7 & 0.8 & 20.8 & 21.4 & & \\
\hline 7218 & HATCREEK & -4.9 & 0.9 & -21.7 & 1.0 & 2.3 & 1.0 & -14.2 & -13.4 & & \\
\hline 7618 & HN-VLBA & 8.1 & 1.3 & -15.0 & 0.7 & -3.1 & 1.0 & 5.9 & -16.9 & & \\
\hline 7242 & HOBART26 & 56.8 & 1.3 & 13.0 & 0.6 & 0.6 & 0.7 & 57.3 & 13.5 & & \\
\hline 7216 & HRAS 085 & -3.6 & 1.1 & -14.1 & 0.8 & 0.0 & 0.6 & -7.6 & -12.8 & & \\
\hline 1856 & KASHIMA & -10.6 & 0.4 & -3.9 & 0.5 & -1.2 & 1.3 & -16.2 & -19.6 & & \\
\hline 1311 & KAUAI & 36.9 & 0.8 & -65.0 & 1.0 & 1.6 & 0.8 & 33.8 & -61.0 & 33.2 & -62.9 \\
\hline 7278 & KODIAK & -14.8 & 1.2 & -16.4 & 1.5 & 1.3 & 5.4 & -22.4 & -8.8 & & \\
\hline 7298 & KOKEE & 36.0 & 0.8 & -64.9 & 1.0 & 2.2 & 0.8 & 33.8 & -61.0 & & \\
\hline 7610 & $\mathrm{KP}-\mathrm{VLBA}$ & -5.4 & 1.0 & -14.3 & 0.9 & 0.2 & 0.9 & -10.6 & -12.5 & & \\
\hline 4968 & KWAJAL26 & 28.4 & 1.6 & -72.2 & 1.5 & -0.6 & 3.5 & 29.4 & -68.3 & 18.6 & -70.8 \\
\hline 7611 & LA-VLBA & -3.5 & 1.1 & -14.8 & 0.8 & -1.0 & 0.6 & -8.5 & -14.2 & & \\
\hline 7310 & MARCUS & 28.4 & 2.5 & -73.8 & 2.6 & 4.6 & 8.0 & 24.6 & -70.1 & & \\
\hline 7243 & MATERA & 19.4 & 0.9 & 24.6 & 1.0 & 0.0 & 0.6 & 13.1 & 22.6 & 23.2 & 18.3 \\
\hline 7230 & MEDICINA & 18.1 & 1.0 & 23.3 & 1.0 & -2.5 & 0.6 & 14.0 & 21.4 & & \\
\hline 7617 & MK-VLBA & 34.8 & 0.9 & -65.6 & 1.1 & -1.9 & 0.9 & 33.6 & -61.2 & & \\
\hline 7222 & MOJAVE12 & -1.1 & 1.0 & -18.4 & 0.9 & -0.7 & 0.5 & -12.5 & -13.0 & -4.7 & -19.0 \\
\hline 7274 & MON PEAK & 16.8 & 1.1 & -45.7 & 1.0 & -4.2 & 2.5 & 23.4 & -42.5 & 17.3 & -42.3 \\
\hline 7612 & NL-VLBA & 1.7 & 1.2 & -16.4 & 0.7 & -4.0 & 0.9 & -2.4 & -16.9 & & \\
\hline 7279 & NOME & -25.3 & 1.5 & -3.0 & 1.7 & 0.0 & 6.4 & -24.0 & -4.5 & & \\
\hline 7547 & NOTO & 20.3 & 1.0 & 22.6 & 1.0 & -1.2 & 0.7 & 21.2 & 20.4 & & \\
\hline 7214 & NRAO85 3 & 4.7 & 1.2 & -14.8 & 0.6 & -1.0 & 0.7 & 2.6 & -15.9 & & \\
\hline 7213 & ONSALA60 & 14.9 & 0.9 & 18.3 & 1.0 & 4.8 & 0.6 & 14.0 & 19.2 & 17.4 & 18.6 \\
\hline 7207 & OVRO 130 & -1.5 & 1.0 & -20.5 & 0.9 & -1.6 & 1.1 & -13.0 & -13.3 & -4.8 & -21.7 \\
\hline 7234 & PIETOWN & -5.6 & 1.0 & -14.9 & 0.9 & -0.8 & 0.7 & -9.2 & -13.6 & & \\
\hline 7256 & PINFLATS & 8.5 & 1.3 & -31.6 & 1.2 & -4.2 & 6.3 & 23.4 & -42.0 & 5.2 & -31.5 \\
\hline 7258 & PLATTVIL & -5.3 & 1.2 & -17.2 & 1.0 & 4.9 & 2.8 & -7.8 & -15.7 & -9.4 & -17.1 \\
\hline 7219 & RICHMOND & 4.8 & 1.1 & -11.6 & 0.6 & -0.6 & 0.7 & 2.4 & -11.4 & 2.7 & -12.8 \\
\hline 1404 & SANTIA12 & 19.2 & 0.7 & 17.9 & 0.6 & 3.6 & 1.5 & 9.8 & -0.9 & & \\
\hline 7227 & SESHAN25 & -13.6 & 0.6 & 31.2 & 0.6 & -0.6 & 1.5 & -13.6 & 22.9 & & \\
\hline 7311 & TSUKUBA & -10.7 & 1.2 & -3.3 & 1.4 & -1.4 & 6.4 & -16.2 & 19.7 & & \\
\hline 7223 & VNDNBERG & 26.0 & 1.0 & -43.7 & 1.0 & 3.0 & 1.1 & 25.1 & -42.0 & 21.6 & -43.6 \\
\hline 7209 & WESTFORD & 7.8 & 1.2 & -16.4 & 0.5 & -0.4 & 0.5 & 6.1 & -16.7 & 6.7 & -18.4 \\
\hline 7224 & WETTZELL & 15.7 & 0.9 & 21.1 & 1.0 & -0.4 & 0.5 & 13.8 & 20.9 & 17.7 & 22.5 \\
\hline
\end{tabular}


Table 2. Kinematic vectors for mobile VLBI tracking sites (in $\mathrm{mm} / \mathrm{yr}$ ).

\begin{tabular}{|c|c|c|c|c|c|c|c|c|c|c|c|}
\hline \multirow{3}{*}{$\begin{array}{l}\text { Station } \\
\text { number }\end{array}$} & \multirow{3}{*}{$\begin{array}{l}\text { Station } \\
\text { name }\end{array}$} & \multicolumn{6}{|c|}{ VLBI } & \multirow{2}{*}{\multicolumn{2}{|c|}{$\begin{array}{c}\text { NUVEL-1 } \\
\text { NNR }\end{array}$}} & \multirow{2}{*}{\multicolumn{2}{|c|}{$\begin{array}{l}\text { Robbins } \\
\text { et al. }\end{array}$}} \\
\hline & & \multicolumn{2}{|c|}{ North } & \multicolumn{2}{|c|}{ East } & \multicolumn{2}{|c|}{ Up } & & & & \\
\hline & & $d x$ & $\sigma_{d x}$ & $d y$ & $\sigma_{d y}$ & $d H$ & $\sigma_{d H}$ & $d x$ & $d y$ & $d x$ & $d y$ \\
\hline 7269 & BLKBUTTE & -6.4 & 1.7 & -20.4 & 1.5 & -7.6 & 9.9 & -12.1 & -12.6 & -7.4 & -19.5 \\
\hline 7286 & ELY & -8.0 & 1.6 & -18.9 & 1.2 & -5.4 & 5.8 & -11.8 & -14.3 & -8.1 & -17.9 \\
\hline 7261 & FLAGSTAF & -6.7 & 1.4 & -15.2 & 1.3 & 12.2 & 6.4 & -10.6 & -13.5 & -8.0 & -17.1 \\
\hline 7266 & FORT ORD & 26.0 & 1.8 & -41.7 & 1.5 & 6.8 & 6.9 & 25.6 & -40.2 & 21.5 & -42.3 \\
\hline 7263 & JPL MV1 & 11.1 & 1.4 & -38.7 & 1.3 & 7.7 & 6.1 & 24.2 & -41.8 & & \\
\hline 1857 & KASHIM34 & -10.9 & 0.6 & -5.3 & 0.6 & -6.4 & 1.5 & -16.2 & 19.6 & -14.7 & -2.7 \\
\hline 7245 & OHIGGINS & 10.8 & 1.7 & 11.8 & 1.3 & 7.7 & 3.8 & 23.5 & 13.1 & & \\
\hline 7254 & PBLOSSOM & 8.4 & 1.4 & -31.9 & 1.4 & -7.6 & 8.6 & -12.9 & -12.6 & 0.2 & -27.4 \\
\hline 7252 & PRESIDIO & 12.9 & 1.2 & -33.6 & 1.2 & -10.6 & 4.2 & -14.5 & -12.8 & 9.5 & -36.4 \\
\hline 7251 & PT REYES & 23.2 & 1.2 & -37.9 & 1.1 & 8.2 & 3.4 & 26.0 & -38.9 & 19.1 & -39.0 \\
\hline 7221 & QUINCY & -3.6 & 1.1 & -24.1 & 1.1 & 5.1 & 3.4 & -14.0 & -13.6 & -3.4 & -20.3 \\
\hline 7602 & TROMSONO & 14.5 & 1.6 & 29.3 & 1.5 & 8.7 & 5.1 & 12.7 & 17.7 & & \\
\hline 7296 & YLOW7296 & -8.6 & 1.0 & -19.0 & 1.0 & 9.2 & 1.7 & -11.6 & -19.5 & & \\
\hline 7894 & YUMA & -6.7 & 1.4 & -15.3 & 1.3 & 14.6 & 6.1 & -11.5 & -12.6 & -7.6 & -13.4 \\
\hline
\end{tabular}

and the Robbins et al. (1993) solution with the predictions of NUVEL-1 NNR model are essentially worse but in the most cases are better than $10 \mathrm{~mm} / \mathrm{yr}$, but we can see for both solutions differences as large as $\sim 20 \mathrm{~mm} / \mathrm{yr}$. It is possible that such large differences are caused by unmodeled movements of particular sites close to the plate boundaries. The residuals of horizontal velocities between our solution and the NUVEL-1 NNR model are the same order as obtained by Heki (1996).

From this comparison we can say that the site velocities are determined more reliably by space tracking data than by geological information. All the more that the lesser difference of our results and those obtained by Robbins et al. (1993) permit us to assume that the KRF can be fixed exclusively using space-based information itself. If it so, it will give for us a way independent of any plate model for fixing KRF.

In any case in the future we must investigate this approach more carefully because it may be possible to look at some new aspects of global and regional plate tectonic processes.

Our numerical experiments indicate that the proposed algorithm is sufficiently robust to the choice and quantity of quasi-stable sites. For example, if we had varied the criterion $|H|<5 \mathrm{~mm} / \mathrm{yr}$ from $5 \mathrm{~mm} / \mathrm{yr}$ to $1 \mathrm{~mm} / \mathrm{yr}$, the alterations of all topocentric velocities changed $<\sim 0.5 \mathrm{~mm} / \mathrm{yr}$ with 18 quasi-stable points instead of 45 .

It should be noted that the fixing of the KRF by a small number of quasi-stable points leads to an increase in the variances of coordinate rates, especially if all points are located on the one area. It also leads to ill conditioning of the normal equations. For example, when we fixed the KRF by 18 sites on the North American plate, the variances of rates increased in 4-5 times compared with Tables 1 and 2.

Most of the listed mobile stations in Table 2 have significantly greater errors than the quasi-stable stations listed in Table 1, especially in vertical movement. It is caused by in- sufficient data. As a whole, half of the vertical movements have a precision better than $1 \mathrm{~mm} / \mathrm{yr}$ so that postglacial rebound might be detected by space-based data.

The precision of horizontal movement is better than the vertical. More than half of stations have $1 \mathrm{~mm} / \mathrm{yr}$ level of error and the remaining are $<2 \mathrm{~mm} / \mathrm{yr}$ level, except for site MARCUS for which the errors in horizontal movements are $<2.6 \mathrm{~mm} / \mathrm{yr}$.

Using an algorithm from Gerasimenko $(1994,1997)$, as a final result we have found that the most likely change of the Earth's radius is $-0.14 \mathrm{~mm} / \mathrm{yr}$ with estimated error \pm 0.18 $\mathrm{mm} / \mathrm{yr}$. The change of the Earth's radius from the changes in the lengths of 340 baselines corresponds to $(0.48 \pm 0.09)$ $\mathrm{mm} / \mathrm{yr}$. The last value was calculated by the algorithm from Gerasimenko (1997). The above obtained values are in a fairly good agreement with the reduction rate of the Earth's radius $(-0.6 \pm 2.5) \mathrm{mm} / \mathrm{yr}$ obtained by Takahashi (1994) and the values $(-0.94 \pm 0.35) \mathrm{mm} / \mathrm{yr}$ and $(-0.01 \pm 0.04) \mathrm{mm} / \mathrm{yr}$ obtained by Lutes (1996). As a whole it is considered that the rate of change of the Earth's radius does not exceed than $1 \mathrm{~mm} / \mathrm{yr}$.

\section{Conclusion}

The approach used in this study to calculate individual station velocities made it possible to look at some new aspects of global and regional tectonic processes. By using only space-based data it is possible to obtain a KRF completely independent from any plate motion model. On the contrary, it is possible to verify such plate model as NUVEL-1 NNR using only geodetic information itself.

The errors in the estimation of station motions were less than $2 \mathrm{~mm} / \mathrm{yr}$ and more than half of stations have $\sim 1 \mathrm{~mm} / \mathrm{yr}$ level of error.

As a final result, we do not find any clear evidence suggesting the change of Earth's radius, which is considerably 
less than $1 \mathrm{~mm} / \mathrm{yr}$.

Acknowledgments. The authors thank Dr. K. Heki (Division of Earth Rotation, National Astronomical Observatory of Japan) for the NUVEL-1 NNR velocities of world-wide VLBI stations and copies of papers that were used in this report.

\section{References}

Argus, D. F. and R. G. Gordon, No-net-rotation model of current plate velocities incorporating, plate motion model NUVEL-1, Geophys. Res. Lett., 18, 2039-2042, 1991.

Fallon, F. W. and W. H. Dillinger, Crustal velocities from geodetic very long baseline interferometry, J. Geophys. Res., 97(B5), 7129-7136, 1992.

Gerasimenko, M. D., Modelling of the change of Earth Dimensions and Deformations from Space Tracking Data, Proc. of the CRCM'93, Kobe, Dec. 6-11, 1993, Special Issue of the J. Geod. Soc. Jap., 215-217, Kyoto, 1994.

Gerasimenko, M. D., A few geodetic arguments in the favour of hypothesis of expanding Earth, Far Eastern Mathematical Reports, 3, 69-79, Vladivostok, 1997.

Heki, K., Horizontal and vertical crustal movements from three-dimensional very long baseline interferometry kinematic reference frame: Implication for the reversal timescale revision, J. Geophys. Res., 101(B2), 3187-3198, 1996.

Lutes, A., Geometrical Analysis of Earth Deformation from VLBI Data, Proc. of the 8th Int. Symp. on Deformation Measurements, 25-28 June 1996, Hong Kong, 309-316, 1996.

Ma, C. and J. W. Ryan, NASA Space Geodesy Program-GSFC DATA Analysis-1998, VLBI Geodetic Results 1979-1998, August, 1998.

Ma, C., J. W. Rayn, and D. S. Caprette, NASA space geodesy programGSFC data analysis-1993, VLBI geodetic results 1979-92, NASA Tech. Memo., 1004605, 1994.

Robbins, J. W., D. E. Smith, and C. Ma, Horizontal crustal deformations and large scale plate motions inferred from space geodetic techniques, in Contributions of Space Geodesy to Geodynamics: Crustal Dynamics, Geodynamics 23, edited by D. E. Smith and D. L. Turcotte, pp. 21-36, AGU, Washington, 1993.

Takahashi, Y., Relation between the station movements by NUVEL-1 model and those observed by VLBI and SLR, J. Geod. Soc. Jap., 40(3), 243-253, 1994.

M. D. Gerasimenko (e-mail: mdger@iam-mail.febras.ru) and T. Kato (e-mail: teru@eri.u-tokyo.ac.jp) 